\title{
Auf den Leim gegangen
}

Wenn etwas kaputt geht, kann man es nähen, nageln, tackern - oder kleben: Das wussten bereits die Steinzeitmenschen vor mehr als 10.000 Jahren und klebten ihre Speerspitzen. Heute werden ganze Flugzeuge geklebt, ja, echte Flugzeuge und Triebwerke. Was bei Flugzeugen funktioniert, kann doch auch in der Medizin nicht schaden, oder?

0 chon im antiken Griechenland war bekannt: wenn nichts mehr hilft, muss auch mal geklebt werden. So schreiben viele Arzneibücher der Frühen Neuzeit, dass bei desperatesten Durchflüssen, oder in Neudeutsch: heftigen Durchfällen, nur Leim helfen könne. In der Regel wurde dazu Tischlerleim empfohlen. Dies ist ein Oberbegriff für Klebstoffe tierischen Ursprungs, wie Knochenleim, Fischleim, Lederleim. Aber Leim ist nicht gleich Leim.

\section{Ochsenleim und Klebkraut}

Kräuterbuchautor Dr. Leonhart Fuchs favorisierte im Jahre 1543 ganz eindeutig den edlen Leim von Ochsenhäuten der Insel Rhodos - vergisst aber auch nicht, zu erwähnen, dass der Ochsenschwanz untauglich sei. Außerdem sollte Leim, mit Essig aufgeweicht, räudige, schuppige Hautstellen heilen und jegliche Art von Hautausschlag vertreiben.

Bei der Herstellung von Leim werden die entsprechenden tierischen Bestandteile aufgekocht. Dabei werden die Kollagenfasern des Bindegewebes herausgelöst und eine gallertige Masse entsteht. Diese ist besonders reich an Glutin, einem Eiweißkörper. Wenn diese Masse weiter gereinigt wird, ist sie auch unter dem Namen Gelatine bekannt. Die Namensähnlichkeit zu „Gluten“, dem Klebereiweiß bezieht sich auf die ähnlichen Eigenschaften, da Gluten den Brotteig ,zusammenklebt“, allerdings ist Gluten rein pflanzlichen Ursprungs. Noch heute wird in Ländern Mittelamerikas "gelatina“" oder Götterspeise als erstes Hausmittel bei Durchfallerkrankungen angewendet. Als
Erklärung ging man in der traditionellen europäischen Medizin davon aus, dass Leim im 4. Grade heiß und trocken sei und daher nach dem Contraria-Prinzip (Gegenmittel-Prinzip) die kalt-feuchten Durchflüsse heilen und stopfen müsse.

Knorpelleim ist ein sehr ähnlicher Stoff, wird allerdings aus hyalinem Knorpel gewonnen und enthält daher im Wesentlichen das Eiweiß Chondrin sowie Mucoproteine. Letztere sind auch Hauptbestandteil von Schleim und der Grund, warum beispielsweise Nasensekret klebrig ist. Knorpelleim wird bis heute sehr erfolgreich als Nahrungsergänzungsmittel bei Arthrose verkauft. Die Arzneibücher unserer Vorfahren hatten aber auch „klebrige“ Rezepte für Veganer im Angebot: Klebkraut, das gegen Ohrenschmerzen helfen und offene Wunden wieder kleben sollte.

\section{Sekundenkleber in der Chirurgie}

Aber auch die moderne Chirurgie setzt Klebstoffe ein. Ein Zwei-Komponentenkleber, ebenfalls auf biologischer Basis, ist Fibrinkleber, der seit den 1970er Jahren bekannt ist. Dieser wird häufig in der Gefäßchirurgie zur Blutstillung, aber auch zum Kleben verletzter Hirnhäute, im HNO-Bereich (z.B. Gehörknöchelchen) sowie bei Face-Liftings eingesetzt. Hierbei werden die natürlichen Eigenschaften der Gerinnungskaskade genutzt. Beim Mischen beider
Komponenten entsteht ein Fibringerinnsel, das die Blutung zum Stillstand bringt.

Gewebekleber dagegen sind künstlich hergestellte Klebstoffe auf Acrylat-Basis. Diese werden aktiviert, sobald sie mit Feuchtigkeit in Verbindung kommen. Einige müssen gekühlt aufbewahrt werden. Sie finden Einsatz bei Schnitt- und Platzwunden als Ersatz der klassischen Naht, aber auch bei der Sklerosierungstherapie von Ösophagusvarizen. Jedoch kommen nur etwa $15-20 \%$ der Wunden für eine solche Klebung in Frage, denn Studien ergaben, dass insbesondere frische, saubere oder chirurgische Wunden von etwa ein bis vier Zentimeter Länge geeignet sind. Ein besonderer Vorteil dieser Kleber ist, dass sie innerhalb weniger Sekunden die Wunde verschließen, einen antibakteriellen Schutzfilm bilden, schmerzfrei aufgetragen werden können und nicht wieder entfernt werden müssen.

DII Dr. Andrea Jessen 